\title{
THE WEB OF QUADRIC HYPERSURFACES IN $r$ DIMENSIONS*
}

\author{
BY T. R. HOLLCROFT
}

1. Introduction. Webs of quadric surfaces were defined and partially investigated in 1862 by de Jonquières. $\dagger$ Later, quadric webs were treated by many mathematicians, among whom were Cremona, Reye, Steiner, and Sturm. $\ddagger$ Still later, the involution defined by a web of quadrics was studied by Snyder and Sharpe. \$ There has been no treatment of webs in a space of higher dimension than three. The purpose of this paper is to derive the properties of a web of algebraic, quadric hypersurfaces in $r$ dimensions.

2. The $W e b$. The equation of a web of quadric hypersurfaces in $S_{r}$ is

$$
\sum \lambda_{i} f_{i}=0, \quad(i=1,2,3,4),
$$

in which the $f_{i}$ are entirely general quadratic functions of $x_{1}, x_{2}, \cdots, x_{r+1}$. The web has a basis manifold $M_{r-4}^{16}$ of order 16 and dimension $r-4$. A doubly infinite non-linear system of quadric hypersurfaces of the web exists such that each hyperquadric of the system has a hypernode, that is, is a quadric hypercone. Also, among the hyperquadrics of the web, there is a doubly infinite non-linear system of pencils of hyperquadrics such that all the hyperquadrics of any one pencil have simple contact at one point. The locus of all such contacts as well as the locus of the hypernodes or vertices of the quadric hypercones of the web, is a surface $J$, the jacobian of the web. The characteristics of $J$ will be found in $\$ 3$.

The web also contains two singly infinite non-linear systems of pencils of hyperquadrics such that all the hyperquadrics in

* Presented to the Society, March 30, 1934.

$\dagger$ Journal de Mathématiques, (2), vol. 7 (1862), p. 412.

$\ddagger$ Pascal, Repertorium der höheren Mathematik, vol. $\mathrm{II}_{2}$ (1922), pp. 629631. Encyklopädie der Mathematischen Wissenschaften, vol. $\mathrm{III}_{2}$, second half, pp. 250-254.

$\S$ Virgil Snyder and F. R. Sharpe, Space involutions defined by a web of quadrics, Transactions of this Society, vol. 19 (1918), pp. 275-290. 
any one pencil have respectively two simple contacts at two points and one stationary contact at one point. The loci of the pairs of contacts and the stationary contacts are, respectively, two curves on $J$. Likewise, the web contains three finite systems of pencils of hyperquadrics such that in any one pencil the hyperquadrics have respectively three simple contacts, one simple and one stationary contact, one four-point contact. These contacts of higher order occur at points of the two curves of $J$. The orders of these curves and the number of hyperquadrics in each of the three finite systems are obtained in $\$ 6$.

Since the equation of a quadric hypersurface in $S_{r}$ contains $r(r+3) / 2$ essential constants and since a hyperplane involves $r$ conditions, $r(r+3) / 2-2 r$ conditions are necessary for a hyperquadric to degenerate into two hyperplanes. Since in the web there are but three conditions at our disposal, for $r(r+3) / 2$ $-2 r>3$, when $r>3$, the web contains no pairs of hyperplanes. For $r=3$, however, as is well known, the web of quadric surfaces contains a finite number, ten, of pairs of planes.

The postulation of a line on a hypersurface of order $n$ in $S_{r}$ is $n+1$, which equals three for $n=2$. The three conditions that a hyperquadric contain a given line are the conditions that the hyperquadric contain three points of the line, which are linear in the coefficients of the hyperquadric. Therefore one and only one hyperquadric of the web is determined by a given line of $S_{r}$.

In determining the singular hyperquadrics of a web, however, we are concerned with the invariant postulation* $I$ of the element on the hyperquadric. The formula for the invariant postulation is $I=P-q$, in which $P$ is the postulation of the element on the hyperquadric and $q$ is the number of conditions necessary to determine the position of the given element in $S_{r}$. For example, in the case of a hypernode on a hyperquadric, we have $P=r+1, q=r$, so that $I=1$. Hence the web of hyperquadrics contains $\infty^{2}$ hyperquadrics with a node, that is, $\infty^{2}$ quadric hypercones of the first species. Since a line is determined in $S_{r}$ by $2(r-1)$ conditions, for a line on a hyperquadric in $S_{r}$, we have $I=3-2(r-1)=-2 r+5$, that is, a hyperquadric of $S_{r}$ contains $\infty^{2 r-5}$ lines.

* T. R. Hollcroft, Invariant postulation, this Bulletin, vol. 36 (1930), pp. 421-426. 
The postulation $P$ of a double line on a hyperquadric of $S_{r}$ is $2 r+1$. Hence, for a double line on a hyperquadric, $I=2 r+1$ $-2(r-1)=3$. The web of hyperquadrics, therefore, contains a finite number of hyperquadrics with a double line, that is, quadric hypercones of the second species. Such a hyperquadric of $S_{r}$ can be projected from the double line into a general hyperquadric of $S_{r-2} .{ }^{*}$ The number of quadric hypercones of the second species contained in the web is given in $\$ 5$.

3. The Jacobian. The jacobian $J$ is the surface that is common to the $r-2$ independent quartic hypersurfaces of the matrix $\left\|\partial f_{i} / \partial x_{j}\right\|,(i=1,2,3,4 ; j=1,2, \cdots, r+1)$; it is a surface of order $n_{0}$, class of plane section $a_{0}$, genus $D_{0}$. It contains $l_{0}$ lines, and has an apparent double curve of order $b_{0}$, which is of class $q_{0}$, and has $t_{0}$ apparent triple points, which are also apparent triple points of $J$. We find for $J$ :

$$
\begin{gathered}
n_{0}=\frac{1}{6} r\left(r^{2}-1\right), \quad a_{0}=\frac{1}{4} r(r-1)\left(r^{2}-r+2\right), \\
D_{0}=\frac{1}{6} r(r-1)(r-2), \quad l_{0}=\frac{1}{6} r(r+1)(r+2), \\
b_{0}=\frac{1}{72} r(r-1)(r-3)(r+4)\left(r^{2}+2\right), \\
2^{3} \cdot 3^{4}\left(q_{0}+4 t_{0}\right)=(r-3)\left(2 r^{8}+6 r^{7}-15 r^{6}-27 r^{5}-129 r^{4}\right. \\
\left.+171 r^{3}-236 r^{2}+12 r-432\right) .
\end{gathered}
$$

For $r=4$, we have $t_{0}=14, q_{0}=120$. The degrees of the expressions in $r$ for $q_{0}$ and $t_{0}$ are 6 and 9 , respectively. Sufficient data were not at hand to determine them separately for any $r$.

The jacobian surface $J$ has no points in common with the basis manifold $M_{r-4}^{16}$ of the web.

4. The Associated Transformation and its Branch-Point Surface. The transformation $\rho y_{i}=f_{i},(i=1,2,3,4)$, associates the hyperquadrics of the web uniquely with the planes of a three-space $(y)$. The web contains $\infty^{4}$ pencils of hyperquadrics, images of the $\infty^{4}$ axial pencils of $(y)$. The image of a line of $(y)$ is the basis manifold $M_{r-2}^{4}$ of the corresponding pencil of hyper-

* E. Bertini, Introduzione alla Geometria Proiettiva degli Iperspazi, 1907, pp. $120-121$. 
quadrics. The manifold $M_{r-2}^{4}$ is of order 4 and class $m_{i}=4(i+1)$, $(i=1, \cdots, r-2)$. The web contains $\infty^{3}$ nets of hyperquadrics, images of the $\infty^{3}$ bundles of planes of $(y)$. The image of a point of $(y)$ is the basis manifold $M_{r-3}^{8}$ of the corresponding net.

The planes of $(y)$ that correspond to quadric hypercones of the first species of the web envelop a surface $L$ in $(y)$ called the branch-point surface. The surfaces $L$ and $J$ are in $(1,1)$ correspondence. The order $N$ of $L$ is the number of intersections of $J$ and two hyperquadrics of the web, so that $N=2 r\left(r^{2}-1\right) / 3$.

To obtain the characteristics of $L$, we must find the characteristics of the branch-point curve $L_{1}$ associated with a net of hyperquadrics in $S_{r}$. These characteristics have been derived in a former paper.* Setting $i=r$ and $n=2$ in the formulas, page 265, there results for the order $n_{1}$, class $m_{1}$, genus $p_{1}$; numbers of nodes $\delta_{1}$, cusps $\kappa_{1}$, bitangents $\tau_{1}$, inflections $\iota_{1}$ of the curve $L_{1}$ :

$$
\begin{aligned}
& n_{1}=r(r+1), \quad m_{1}=r+1, \quad p_{1}=\frac{1}{2}(r+1)(r-2)+1, \\
& \delta_{1}=\frac{1}{2}\left(r^{2}-1\right)(r-2)(r+4), \quad \kappa_{1}=3\left(r^{2}-1\right), \quad \tau_{1}=\iota_{1}=0 .
\end{aligned}
$$

As has been proved in a former paper, $\uparrow$ these are the characteristics of a plane section of the tangent cone of $L$.

The characteristics of $L$ will be represented by these symbols:

$N\left[n^{\prime}\right]$ order [class];

$a\left[a^{\prime}\right]$ order of tangent cone [class of plane section];

$\kappa^{\prime}\left[\delta^{\prime}\right]$ number of inflections [bitangents] of plane section;

$\kappa[\delta]$ number of cuspidal [nodal] lines of tangent cone;

$b[c]$ order of nodal [cuspidal] curve;

$b^{\prime}\left[c^{\prime}\right]$ class of bitangential [spinodal] developable;

$q\left[r^{\prime}\right]$ class of nodal [cuspidal] curve;

$\beta[\gamma](i)$ number of intersections of nodal and cuspidal curves which are cusps on cuspidal [nodal] (neither) curve;

$\beta^{\prime}\left[\gamma^{\prime}\right]\left(i^{\prime}\right)$ number of common planes of bitangential and spinodal developables which are stationary on the spinodal [bitangential] (neither) developable;

$t\left[t^{\prime}\right]$ number of triple points [planes] of nodal curve [bitangential developable];

* T. R. Hollcroft, Nets of manifolds in i dimensions, Annali di Matematica, (4), vol. 5 (1927-28), pp. 261-267.

$\dagger$ T. R. Hollcroft, Transactions of this Society, vol. 35 (1933), p. 859. 
$\rho^{\prime}\left[\sigma^{\prime}\right]$ order of bitangential [spinodal] curve;

$\rho[\sigma]$ class of nodal [cuspidal] developable;

$C^{\prime} \quad$ number of conic tropes;

$D$ genus.

Since the characteristics of the tangent cone are known and since from $b^{\prime}=c^{\prime}=0$, we obtain $\sigma^{\prime}=\rho^{\prime}=\beta^{\prime}=\gamma^{\prime}=t^{\prime}=0$; the remaining characteristics of $L$ are computed by means of the Cayley-Zeuthen equations relating the characteristics of reciprocal surfaces. There results:

$$
\begin{aligned}
N & =\frac{2}{3} r\left(r^{2}-1\right), \quad a=a^{\prime}=r(r+1), \quad n^{\prime}=r+1, \\
\delta & =\frac{1}{2}\left(r^{2}-1\right)(r-2)(r+4), \quad \kappa=3\left(r^{2}-1\right), \\
\delta^{\prime} & =\frac{1}{6} r(r+1)\left(3 r^{2}-8 r+8\right), \quad \kappa^{\prime}=r\left(r^{2}-1\right), \\
b & =\frac{1}{18} r(r+1)\left[4 r(r-1)\left(r^{2}-1\right)-87 r+159\right], \\
c & =3 r(r+1)(r-2), \\
\rho & =\frac{1}{3}(r+1)\left(2 r^{4}-2 r^{2}-39 r+33\right), \\
\beta & =2(r+1)\left(9 r^{2}-28 r+13\right), \quad i=i^{\prime}=0, \\
\gamma & =2(r+1)\left[r^{2}\left(r^{2}-1\right)(r-2)-39 r^{2}+114 r-48\right], \\
t & =\frac{1}{81}(r+1)\left(4 r^{8}-4 r^{7}-8 r^{6}-253 r^{5}+481 r^{4}+257 r^{3}\right. \\
q & =\frac{1}{3}(r+1)\left(2 r^{4}-29 r^{2}+45 r-6\right), \\
r^{\prime} & =(r+1)\left(5 r^{2}-10 r+2\right), \quad C^{\prime}=\frac{1}{6} r(r+1)(r+2), \\
D & =\frac{1}{6} r(r-1)(r-2) .
\end{aligned}
$$

The image of $L$ is a hypersurface $R$ in $(x)$ of order $4 r\left(r^{2}-1\right) / 3$ which contains the jacobian surface $J$ as a double surface.

5. The Quadric Hypercones of the Second Species. To the $r(r+1)(r+2) / 6$ conic tropes of $L$ correspond uniquely $r(r+1)(r+2) / 6$ lines of $J$. To the plane which has contact with 
$L$ along a conic, forming a conic trope, corresponds a hyperquadric with the corresponding line of $J$ as a double line, that is, a quadric hypercone of the second species. The web of hyperquadrics, therefore, contains $r(r+1)(r+2) / 6$ quadric hypercones of the second species. The contact conic on $L$ and the corresponding line on $J$ are each the image of the other. For $r=3$, the $r(r+1)(r+2) / 6$ quadric hypercones of the second species are ten pairs of planes.

6. Loci of Contacts. As has been stated, $J$ is the locus of simple contacts of hyperquadrics of the web; $J$ also contains all contacts of higher order.

To the cuspidal curve $c$ of $L$ corresponds a binodal manifold $c_{r-2}$ of $R$ of dimension $r-2$ and order $12 r(r+1)(r-2)$. This manifold $c_{r-2}$ and $J$ both lie on $R$ and intersect in a curve $c_{1}$ of order $3 r(r+1)(r-2) / 2$. The curve $c_{1}$ is a contact curve of $J$ and $c_{r-2}$. The points of $c$ and $c_{1}$ are in $(1,1)$ correspondence. The curve $c_{1}$ is the locus of the points of stationary contact of pencils of hyperquadrics of the web.

To the nodal curve $b$ of $L$ corresponds a nodal manifold $b_{r-2}$ of $R$ of dimensions $r-2$ and order

$$
2 r(r+1)\left[4 r(r-1)\left(r^{2}-1\right)-87 r+159\right] / 9 .
$$

The surfaces $J$ and $b_{r-2}$ intersect in a curve $b_{1}$ whose order equals that of $b$. The image of $b_{1}$ is $b$ counted twice. The points of $b$ and $b_{1}$ are in $(1,2)$ correspondence. The curve $b_{1}$ is the locus of pairs of contacts of pencils of hyperquadrics of the web.

If $P$ is a point $\gamma$ of $L$, at which $b$ and $c$ intersect and $b$ has a cusp, the image of $P$ on $J$ consists of two points, $P_{1}$ at an intersection of $b_{1}$ and $c_{1}$, and $P_{2}$ on $b_{1}$ only. The hyperquadrics of a pencil of the web have stationary contact at $P_{1}$ and simple contact at $P_{2}$. Since $L$ has $\gamma$ such points, the number of pencils of hyperquadrics of the web that have one stationary and one simple contact is the value of $\gamma$ given in $\$ 4$.

If $P$ is a point $\beta$ of $L$ at which $b$ and $c$ intersect and $c$ has a cusp, the image of $P$ on $J$ is a point $P_{0}$ which lies at a contact of $b_{1}$ and $c_{1}$. The hyperquadrics of a pencil of the web have 4-point contact at $P_{0}$. Since $L$ has $\beta$ such points, the number of pencils of hyperquadrics of the web that have 4-point contact is the value of $\beta$ given in $\S 4$. 
If $P$ is a triple point $t$ of $b$ on $L, P$ corresponds to three points of $b_{1}$. At these points, the hyperquadrics of three pencils, images of the three tangents to $b$ at $P$, have simple contact. Hence the number of pencils of hyperquadrics of the web that have three simple contacts is $3 t$, in which $t$ has the value given in $\$ 4$.

7. Basis Elements. As stated in $\$ 2$, the web has a basis manifold of order 16 and dimension $r-4$. The web may have special basis elements which are equivalent to a part of the basis manifold $M_{r-4}^{16}$. A web of proper hyperquadrics can not have multiple basis elements.

In $S_{3}$, a web of quadrics with basis points is a special web. For $r=4$ the general web has 16 points, and for $r>4$ it has an infinite number contained in $M_{r-4}^{16}$.

For $r \leqq 4$, webs with basis 1 nes are highly special. In $S_{5}$ a basis line becomes part of the basis curve and for $r \geqq 6$, the basis line lies on $M_{r-4}^{16}$. Similarly for planes and elements of higher dimension. Webs of hyperquadrics may also have basis curves, surfaces, etc., of given order forming a part of or lying on $M_{r-4}^{16}$.

The basis elements $S_{0}, S_{1}, S_{2}, \ldots, S_{\alpha}$ have the respective postulation $1,3,6, \cdots,(\alpha+1)(\alpha+2) / 2$ on a hyperquadric in $S_{r}$.

Since a web must contain three and only three arbitrary parameters, in establishing a web of hyperquadrics in $S_{r}$ there are $r(r+3) / 2-3$ constants at our disposal. For $r=3$, this number is 6 , that is, a web may have a maximum of 6 basis points or 2 basis lines. For $r=4$, this number is 11 , that is, a web in $S_{4}$ may have 1, 2 or 3 basis lines in which cases it has also 11, 6, 1 basis points, respectively. In $S_{r}$, finally, the maximum number of basis lines in a web of hyperquadrics is the largest integer contained in $\left(r^{2}+3 r-6\right) / 6$.

Similarly, a web of quadric hypersurfaces in $S_{4}$ may have one and only one basis plane, in which case it has in addition 5 basis points. The maximum number of basis planes in a web of quadric hypersurfaces in $S_{r}$ is the largest integer contained in $\left(r^{2}+3 r-6\right) / 12$.

Finally, the maximum number of basis $S_{\alpha}$ 's that can be assigned to a web of quadric hypersurfaces in $S_{r}$ is the largest integer contained in $\left(r^{2}+3 r-6\right) /[(\alpha+1)(\alpha+2)]$.

Wells College 